\title{
ARTÍCULO ORIGINAL \\ Uso de ayudas diagnósticas en el paciente intoxicado en el servicio de urgencias
}

Fecha de recibido:

26 de mayo de 2020.

Fecha de aprobación: 25 de agosto de 2020 .
Forma de citar este artículo: Caicedo LG, Rodríguez ND, Urrego JC, Berrouet MC, Massaro MM, Valencia NL. Uso de ayudas diagnósticas en el paciente intoxicado en el servicio de urgencias. Med UPB. 2021;40(1):28-34. DOI:10.18566/medupb.v40n1.a05

1 Medicina de urgencias, Universidad CES. Medellín, Colombia.

2 Toxicología clínica, Universidad CES. Medellín, Colombia.

3 Investigación e innovación, Universidad CES. Medellín, Colombia.

4 Gerencia de sistemas de información en salud, Universidad CES. Medellín, Colombia.

Dirección de correspondencia: Nelcy Lorena Valencia Ortiz. Correo electrónico: lorenavalenciaortiz@gmail. com

\section{Use of diagnostic aids in the poisoned patient in the emergency department / Uso de ajudas diagnósticas em paciente intoxicado no atendimento de emergência}

Luis Gabriel Caicedo Bello ${ }^{1}$, Nelson Darío Rodríguez Flórez ${ }^{1}$, Juan Camilo Urrego Sepúlveda ${ }^{1}$, Marie Claire Berrouet Mejía ${ }^{2}$, Mónica María Massaro Ceballos ${ }^{3}$, Nelcy Lorena Valencia Ortiz ${ }^{4}$

\section{RESUMEN}

Objetivo: La incidencia de intoxicaciones en el servicio de urgencias viene en aumento en Colombia. En el abordaje inicial se solicitan paraclínicos de rutina, en ocasiones sin una correlación entre el xenobiótico, la fisiopatología y el riesgo clínico. El objetivo de esta investigación es describir el uso de las ayudas diagnósticas en el paciente intoxicado en el servicio de urgencias de un hospital de alta complejidad.

Metodología: Estudio descriptivo transversal con disponibilidad de datos para el periodo 2014-2016. Se recopiló información de los registros de las historias clínica de los pacientes que acudieron al servicio de urgencias con diagnósticos relacionados con intoxicaciones. Resultados: El 55.4\% de la población fue de sexo femenino, el 54.5\% eran menores de 25 años y la mayoría pertenencia al área urbana (77.7\%). La intencionalidad en el $68.9 \%$ fue suicida. Se reportó el toxídrome en el $17.6 \%$ de los casos, donde el sedante fue el más común (9.2\%). Las sustancias más usadas fueron los psicofármacos (22.8\%), siendo los antidepresivos tricíclicos más comunes con un $10.9 \%$; seguidos de los plaguicidas (19.1\%), donde los organofosforados fueron los más comunes (8.8\%). Las ayudas diagnósticas más solicitadas fueron el hemograma en un $94.3 \%$ de los casos, seguido de la creatinina en un $90.2 \%$. El electrocardiograma se realizó en el $49.7 \%$ de los casos y los tóxicos en orina en el $7.8 \%$.

Conclusión: Se evidencia un uso rutinario de ayudas diagnósticas en el servicio de urgencias; se requieren estudios adicionales que evalúen la pertinencia de ayudas diagnósticas en este escenario.

Palabras clave: diagnóstico; urgencias; intoxicación

\section{ABSTRACT}

Objective: The incidence of poisoning in the emergency department is increasing in Colombia. In the initial approach, paraclinical routines are requested, sometimes without a correlation between xenobiotic, pathophysiology and clinical risk. The objective of this research is to describe the use of diagnostic aids in poisoned patients in the emergency department of a high-complexity hospital.

Methodology: Descriptive cross-sectional study with retrospective data availability for the 2014-2016 period. Information was collected from the medical records of patients who came to the emergency department with diagnoses related to poisoning. Results: $55.4 \%$ of the population was female, $54.5 \%$ were under the age of 25 , the majority belonging to the urban area (77\%). The intentionality in $68.9 \%$ of them was suicidal. Toxidrome was reported in $17.6 \%$ of cases, the sedative being the most common $(9.2 \%)$. Psychotropic drugs were the most commonly used substances (22.8\%), the tricyclic antidepressant being the most used with $10.9 \%$, followed by pesticides with $19.1 \%$, among 
which organophosphates were the most common (8.8\%). The most requested diagnostic aids were the hemogram in $94.3 \%$ of the cases, followed by creatinine in $90.2 \%$. The electrocardiogram was performed in $49.7 \%$ of the cases and the toxic in urine in $7.8 \%$. Conclusions: A routine use of diagnostic aids is evidenced in the emergency department. Therefore, additional studies are required to evaluate the relevance of diagnostic aids in this setting.

Keywords: diagnosis; emergencies; poisoning

\section{RESUMO}

Objetivo: A incidência de intoxicações no setor de emergência está aumentando na Colômbia. Na abordagem inicial, são solicitadas paraclínicas de rotina, às vezes sem correlação entre o xenobiótico, a fisiopatologia e o risco clínico. O objetivo desta pesquisa é descrever a utilização de meios auxiliares de diagnóstico em pacientes intoxicados no pronto-socorro de um hospital de alta complexidade.

Metodologia: Estudo transversal descritivo com disponibilidade de dados para o período 2014-2016. As informações foram coletadas nos prontuários dos pacientes que compareceram ao pronto-socorro com diagnóstico de intoxicação.

Resultados: $55,4 \%$ da população era do sexo feminino, 54,5\% tinham menos de 25 anos e a maioria pertencia à zona urbana (77,7\%). A intencionalidade em $68,9 \%$ foi suicida. Toxidromia foi relatada em $17,6 \%$ dos casos, sendo o sedativo o mais comum $(9,2 \%)$. As substâncias mais utilizadas foram os psicotrópicos (22,8\%), sendo os antidepressivos tricíclicos mais comuns com 10,9\%; seguido por agrotóxicos (19,1\%), onde os organofosforados foram os mais comuns ( $8,8 \%$ ). Os meios diagnósticos mais solicitados foram o hemograma em $94,3 \%$ dos casos, seguido da creatinina em $90,2 \%$. O eletrocardiograma foi realizado em $49,7 \%$ dos casos e a urina tóxica em $7,8 \%$.

Conclusão: Evidencia-se o uso rotineiro de meios auxiliares de diagnóstico no prontosocorro; Estudos adicionais são necessários para avaliar a relevância dos auxiliares de diagnóstico neste cenário.

Palavras-chave: diagnóstico; emergências; envenenamento

\section{INTRODUCCIÓN}

Las intoxicaciones son una causa frecuente de ingreso al servicio de urgencias. En Estados Unidos, el centro de control de intoxicaciones (AAPCC (por sus siglas en inglés) reporto 2159032 millones de llamadas asociadas a esa causa durante $2016^{1}$.

Según reportes del Sistema Nacional de Vigilancia en Salud Pública (Sivigila), en 2018 en Colombia se notificaron 28094 casos de intoxicación, donde en primer lugar lo ocuparon las sustancias psicoactivas (32.1\%), seguido de los accidentes ofídicos $(18.8 \%)$ y por medicamentos $(15.2 \%)^{2}$. Hasta la semana epidemiológica 14 de 2019 se habían reportado 3828 casos, con las sustancias psicoactivas en primer lugar (30.2\%). En los años 2015-2017 se han reportado 33 787, 36003 y 39709 casos, donde la primera causa fueron medicamentos, seguido por plaguicidas. La incidencia actual es de 15.5 casos por cada 100000 habitantes, con un aumento gradual en la cantidad de eventos ${ }^{3,4}$.

En Colombia se han desarrollado guías de atención médica y existen líneas de asesoría nacional en las que se sugiere un abordaje inicial y qué ayudas diagnósticas realizar. Sin embargo, si no se individualizan las decisiones según cada paciente, se corre el riesgo de pedir ayudas diagnósticas que no cambian el pronóstico, ni el tratamiento y que, por el contrario, retrasan el manejo definitivo mientras se esperan los resultados.

En el abordaje de un paciente intoxicado, la anamnesis, el examen físico y el conocimiento del agente agresor y de sus efectos pudieran ser suficientes en muchos casos. El uso de ayudas diagnósticas de manera indiscriminada ocasiona confu- 
sión y conductas inadecuadas. Este es el caso del dímero $\mathrm{D}$ en accidentes ofídicos, que puede estar elevado en pacientes con edema y eritema en las extremidades y llevar a pensar erróneamente en trombosis venosa profunda. En el sentido contrario, como paraclínico de mayor utilidad está el electrocardiograma (EKG) que permite aclarar los diagnósticos y tomar decisiones en el servicio de urgencias. El resto de paraclínicos se deben racionalizar según las circunstancias individuales ${ }^{5,6}$.

La mayoría de los pacientes que consultan a urgencias por intoxicación requieren intervenciones de soporte vital básico y avanzado, con protocolos como de soporte avanzado de la vida en el trauma (ATLS por sus siglas en inglés $)^{7}$ y teniendo en mente que el uso de ayudas diagnósticas no debe poner en riesgo la vida del paciente ni retrasar la toma de decisiones específicas ${ }^{8}$.

Entre las ayudas diagnósticas, cabe mencionar las pruebas rápidas para tóxicos comúnmente denominadas tóxicos en orina que tienen poca utilidad en urgencias dado que no informan la dosis ni el tiempo trascurrido desde el consumo y tienen múltiples falsos positivos (hasta un $21.5 \%)^{9}$. Además, si no se interpretan de manera adecuada, generan confusión y llevan a tomar decisiones incorrectas. Un ejemplo es el resultado positivo de cannabis, que podría explicarse por un consumo en los últimos 30 días o por un falso positivo por consumo de antiinflamatorios no esteroides (AINE), como el ibuprofeno o naproxeno, o por consumo de inhibidores de la bomba de protones (IBP), como el omeprazol ${ }^{10}$.

Al buscar información en Colombia, se evidencia un vacío en el conocimiento sobre el uso de ayudas diagnósticas y su aplicación en el paciente intoxicado. El objetivo de esta investigación fue realizar una aproximación al describir el uso de las ayudas diagnósticas en el paciente intoxicado en el servicio de urgencias de una institución de alta complejidad, iniciando con la caracterización de las más utilizadas y a partir de la descripción de información para que en futuros estudios se evalué la pertinencia y el uso de las ayudas diagnósticas.

\section{METODOLOGÍA}

Se realizó un estudio descriptivo transversal con datos obtenidos a partir de las historias clínicas. La población estuvo conformada por todos los pacientes que ingresaron en el servicio de urgencias de una institución de alta complejidad de Medellín entre el 1 de enero de 2014 y el 31 de diciembre de 2016 ( $n=193)$ a quienes se les hizo diagnóstico de intoxicación por algún xenobiótico. Se utilizaron los diagnósticos de egreso CIE-10 T36 a T65, las fichas epidemiológicas y bases de datos del servicio de toxicología.

Se diseñó un instrumento de recolección en el programa Excel 2016 con las variables sociodemográficas, clíni- cas y paraclínicas de interés. Su diligenciamiento estuvo a cargo de tres médicos residentes de la Especialización en Medicina de Urgencias, entrenados en la recolección de la información. El control de calidad del instrumento se realizó durante y después de diligenciada la información $y$, como resultado de este proceso, se eliminaron registros en los que no se reportara en las historias clínicas el respectivo código CIE-10 o que contuvieran diagnósticos sin relación con intoxicaciones.

Para el análisis estadístico se calcularon frecuencias absolutas y relativas para las variables cualitativas y las variables cuantitativas medidas de tendencia central y dispersión. El análisis se realizó en SPSS 21.

\section{RESULTADOS}

Se analizaron 193 historias clínicas. El 55.4\% de la población era de sexo femenino. En relación con la edad, el 54.5\% eran menores de 25 años. Al valorar otras características sociodemográficas, como el lugar de procedencia, el $77.7 \%$ pertenecía al área urbana, el $82.2 \%$ no tenía pareja estable (separado, soltero o viudo), el $57.6 \%$ se encontraba en condición de desempleo y la escolaridad de la mayoría era primaria (44.7\%). En la Tabla 1 se presentan todas las características.

La intencionalidad de la intoxicación en el $68.9 \%$ de la población fue el intento suicida, seguida en un $14.5 \%$ de causa accidental o iatrogénica, y en un $4.7 \%$ por otras causas, entre las que se encuentran cuatro casos con fines delictivos. En la historia clínica solo se registró la presencia de un toxídrome específico en el 17.6\% de los casos, donde el más frecuente fue el sedante con un 9.2\%. En el $82.4 \%$ de los casos la información del toxídrome estaba incompleta o no se registraba. El promedio de estancia en urgencias fue de 2.3 días (DE 1.36) con un máximo de nueve días de estancia.

En cuanto a la disposición final del paciente, del 53.9\% de los casos egreso del servicio de urgencias, el $23.8 \%$ se hospitalizó en salas generales. El porcentaje de pacientes que se manejó en unidades de alta dependencia (UCI/ UCE) fue del $17.1 \%$.

Con respecto a la etiología de las intoxicaciones los xenobióticos más reportados fueron psicofármacos con un $22.8 \%$, y entre ellos los principales los antidepresivos tricíclicos con un $10.9 \%$, seguido de benzodiacepinas con un 3.6\%. El siguiente grupo fue el de plaguicidas con un $19.1 \%$, entre ellos los más comunes fueron los organofosforados con un $88 \%$, seguido del flouracetato de sodio (FAS) con un 4.1\%. Dentro de los analgésicos el más común fue acetaminofén con un $18 \%$. Con respecto a las drogas de abuso, las más comunes fueron las sustancias estimulantes con un $7.8 \%$ donde se encuentra la cocaína en un $7.3 \%$. Otras sustancias de abuso reportadas fueron el alcohol etílico en un 5.7\% de los casos; 
Tabla 1. Características sociodemográficas y clínicas de ingreso de los pacientes intoxicados en un servicio de urgencias $(n=193)$.

\begin{tabular}{|c|c|c|c|}
\hline Variables & & $\mathbf{n}$ & $\%$ \\
\hline \multirow{2}{*}{ Sexo } & Hombre & 86 & 44.6 \\
\hline & Mujer & 107 & 55.4 \\
\hline \multirow{4}{*}{ Edad } & Menores de 12 años & 14 & 7.3 \\
\hline & $13-25$ años & 91 & 47.2 \\
\hline & 26-40 años & 55 & 28.5 \\
\hline & Mayores de 40 años & 33 & 17.1 \\
\hline \multirow{4}{*}{ Escolaridad } & Ninguna & 17 & 16.5 \\
\hline & Primaria & 46 & 44.7 \\
\hline & Bachiller & 23 & 22.3 \\
\hline & Superior & 17 & 16.5 \\
\hline \multirow{2}{*}{ Estado civil } & Con pareja & 32 & 17.8 \\
\hline & Sin pareja & 148 & 82.2 \\
\hline \multirow{2}{*}{ Condición de empleo } & Empleado & 70 & 42.4 \\
\hline & Desempleado & 95 & 57.6 \\
\hline \multirow{2}{*}{ Área de residencia } & Urbano & 150 & 77.7 \\
\hline & Rural & 42 & 21.8 \\
\hline \multirow{4}{*}{ Intencionalidad } & Accidental/Iatrogénica & 28 & 14.5 \\
\hline & Recreacional & 23 & 11.9 \\
\hline & Suicida & 133 & 68.9 \\
\hline & Otra & 9 & 4.7 \\
\hline \multirow{7}{*}{ Toxidrome } & Adrenérgico & 3 & 1.6 \\
\hline & Anticolinérgico & 4 & 2.1 \\
\hline & Colinérgico & 4 & 2.1 \\
\hline & Opioides & 2 & 1 \\
\hline & Sedante & 18 & 9.3 \\
\hline & Serotoninérgico & 3 & 1.6 \\
\hline & Sin información & 159 & 82.4 \\
\hline
\end{tabular}

el tetrahidrocanabinol (THC) se encontró en un 4.1\%. En cuanto a los casos asociados a tóxicos, el accidente ofídico bothrópico se presentó en un 4.7\%,y de los casos relacionados con productos para la limpieza, el producto más común fue el hipoclorito con un $4.1 \%$.

Con relación a las ayudas diagnósticas solicitadas, la más común fue el hemograma en un $94.3 \%$, seguido del ionograma donde electrolitos, como el potasio, se solicitaron en el $81.3 \%$, el sodio en el $80.3 \%$ y el cloro en el $58.5 \%$. La tercera ayuda más común fueron las pruebas renales, se pidió creatinina en el $90.2 \%$ de los casos y nitrógeno ureico (BUN) en un $73.6 \%$. Las pruebas de integralidad hepática, transaminasas (AST/ALT), se solicitaron en el $49.2 \%$ y los gases arteriales en un $46.6 \%$ de la población.
E1 EKG se realizó en el 49.7\% de la población y los tóxicos en orina en el 7.8\%. Entre otras ayudas diagnósticas solicitadas se encontró la proteína C-reactiva (PCR) en el 18.7\%, la gamma-glutamil transferasa (GGT) en el 5.7\%, la fosfatasa alcalina en el $19.2 \%$ y lactato deshidrogenasa $(\mathrm{LDH})$ en el $4.7 \%$.

$\mathrm{Al}$ relacionar las etiologías de la toxicidad y las ayudas diagnósticas solicitadas (Tabla 2), se evidenció que para los antidepresivos tricíclicos las más utilizadas fueron el hemograma y la creatinina en un $95.2 \%$, seguido de sodio, potasio y BUN en el $90.5 \%$, y con un menor porcentaje el EKG en el 85.7\%. Los tóxicos en orina se solicitaron en el 19\% de los casos. En las intoxicaciones por antipsicóticos el EKG y los tóxicos en orina se solicitaron en el 100\% de los casos, seguido de sodio, potasio, creatinina y el 
Tabla 2. Ayudas diagnósticas según sustancias en pacientes intoxicados en un servicio de urgencias.

\begin{tabular}{lcccccccccc}
\hline \multirow{2}{*}{ Ayudas } & \multicolumn{10}{c}{ Sustancias } \\
\cline { 2 - 12 } & \multicolumn{1}{c}{ Tricíclicos } & \multicolumn{10}{c}{ Benzodiacepinas } & \multicolumn{1}{c}{ Antipsicóticos } & \multicolumn{2}{c}{ Organofosforados } & \multicolumn{2}{c}{ Cocaína/Derivados } \\
\cline { 2 - 12 } & $\mathbf{n}$ & $\%$ & $\mathbf{n}$ & $\%$ & $\mathbf{n}$ & $\%$ & $\mathbf{n}$ & $\%$ & $\mathbf{n}$ & $\%$ \\
\hline EKG & 18 & 85.7 & 4 & 57.1 & 5 & 100 & 11 & 64.7 & 6 & 42.9 \\
Sodio & 19 & 90.5 & 6 & 85.7 & 4 & 80 & 15 & 88.2 & 11 & 78.6 \\
Potasio & 19 & 90.5 & 6 & 85.7 & 4 & 80 & 15 & 88.2 & 12 & 85.7 \\
Creatinina & 20 & 95.2 & 6 & 85.7 & 4 & 80 & 17 & 100 & 13 & 92.9 \\
BUN & 19 & 90.5 & 5 & 71.4 & 4 & 80 & 13 & 76.5 & 11 & 78.6 \\
Transaminasas & 15 & 71.4 & 4 & 57.1 & 2 & 40 & 8 & 47.1 & 3 & 21.4 \\
Tóxicos en orina & 4 & 19.0 & 2 & 28.6 & 5 & 100 & 17 & 100 & 3 & 21.4 \\
Gases & 14 & 66.7 & 3 & 42.9 & 2 & 40 & 9 & 52.9 & 3 & 21.4 \\
Hemograma & 20 & 95.2 & 6 & 85.7 & 3 & 60 & 17 & 100 & 14 & 100 \\
\hline
\end{tabular}

BUN en el 80\%, y el hemograma en el 60\% de los casos. Para plaguicidas como los inhibidores de la colinesterasa se encontró que el hemograma, los tóxicos en orina y la creatinina se solicitaron en el $100 \%$ de los casos, seguido de sodio y potasio en el 88.2\%, y el EKG en el $64.7 \%$.

\section{DISCUSIÓN}

El presente estudio evidenció que la principal causa de intoxicación fue por medicamentos, específicamente psicofármacos en un $22 \%$ de los casos. Este resultado difiere con los hallazgos del estudio de Estrada et al. (2018) donde la primera causa fueron los plaguicidas. La intencionalidad en el citado estudio: en un $60.2 \%$ suicida y en el presente en el 68.9\%. En ambos estudios no tener pareja y la condición de desempleo fueron comunes en esta intencionalidad ${ }^{11}$.

Llamativamente, la intoxicación por estimulantes solo fue encontrada en un $7.8 \%$, lo que difiere del reporte nacional del Sivigila 2019 (semana epidemiológica 34), donde la primera causa de intoxicación fueron los estimulantes hasta en el $37.1 \%{ }^{12}$. Estudios en otras ciudades del país, como el de Terán et al. en 316 pacientes en Montería, reportan como primera causa de intoxicación los plaguicidas (28.7\%), seguidos del accidente ofídico (24.3\%). Estas diferencias pueden ser atribuidas al tipo de población, ya que el $64.5 \%$ de los casos de ese reporte procedía de área rural $^{13}$, mientras que en el presente estudio era minoría.

Las intoxicaciones secundarias a abuso de sustancias, bien sea por alcohol etílico, cannabis o cocaína, u otros estimulantes, no llegaron al 30\% de la muestra, hallazgo contrario a lo reportado en Europa donde la mayoría de los tóxicos son el alcohol etílico (58-75\%) ${ }^{14,15}$, seguido de los antidepresivos en un 17\%. Esta diferencia pudiera explicarse por diferencias sociodemográficas y culturales, así como por la presencia de estaciones en Europa, que puede tener repercusiones en el comportamiento de los eventos de salud. Estas diferencias son una limitante al comparar estrategias diagnósticas y terapéuticas ${ }^{16}$.

El toxídrome más común fue el sedante en el 9.2\% de los pacientes, sin embargo, el dato más llamativo es que en el $82.4 \%$ de las historias clínicas revisadas este no solo no se reportaba, sino que además no había la suficiente información para deducirlo. Es importante resealtar que la clasificación por toxídrome es una herramienta valiosa en el enfoque inicial, que solo necesita de un examen físico acucioso para ayudar en la toma de decisiones sobre ayudas diagnósticas y medidas terapéuticas ${ }^{17}$.

El hemograma en un $95 \%$, seguido de iones y función renal con un $90.5 \%$, fueron las ayudas diagnósticas más utilizadas, independiente del tipo de intoxicación. Se realizaron además estudios como la PCR en alrededor del $18 \%$ de los pacientes y otros como LDH, GGT en cerca del $5 \%$, que no son relevantes en la toma de decisiones durante el enfoque inicial ${ }^{17,18}$.

$\mathrm{Al}$ relacionar las ayudas diagnósticas con las etiologías de las intoxicaciones, se encuentra que hasta el 15\% de los pacientes intoxicados por antidepresivos tricíclicos no tenían EKG, necesario para definir intervenciones críticas en urgencias, mientras que otros estudios, como el hemograma y el nitrógeno ureico, fueron más frecuentes que el EKG a pesar de ser poco útiles para la toma de decisiones en este escenario ${ }^{6,17}$.

La valoración paraclínica de drogas de abuso o "tóxicos en orina", si bien globalmente fue utilizada en el $7.8 \%$, se solicitó en el 100\% de las intoxicaciones por organofosforados y antipsicóticos, y en el 19\% de las intoxicaciones por antidepresivos tricíclicos. Sin embargo, por historia clínica y hallazgos al examen físico, ya se había hecho una identificación de la sustancia, lo que en este contexto es innecesaria la petición de esta prueba que incluso pudiera 
aumentar la estancia en urgencias. Los tóxicos en orina tienen gran incidencia de falsos positivos y negativos, que, si se interpretan sin un contexto clínico adecuado, confunden la toma de decisiones ${ }^{18}$.

En resumen, según los datos del estudio, a la mayoría de pacientes se les solicita ayudas diagnósticas de modo rutinario, sin considerar el proceso fisiopatológico de la sustancia tóxica. Estos hallazgos, que son congruentes con otros estudios como el de Terán et al. ${ }^{13}$, implican incremento en los costos de la atención y en la estancia de los pacientes en los servicios de urgencias, lo que favorece la sobreocupación de urgencias e interfiere con la toma de decisiones críticas en el abordaje de la intoxicación aguda ${ }^{19}$.

Dentro de las limitaciones del estudio hay que mencionar que al recolectar información de las historias clínicas se pueden presentar subregistros o errores u omisiones en el registro de algunas enfermedades.

En conclusión, con este trabajo se aporta al conocimiento del uso de ayudas diagnósticas en el paciente intoxicado en urgencias. Los resultados pueden extrapolarse a instituciones de características similares. Si bien el objetivo es describir las ayudas utilizadas, con los datos encontrados se puede inferir que hay un uso rutinario e inapropiado de estas, sin considerar los mecanismos fisiopatológicos del agente tóxico. Hay que considerar que este trabajo se realizó en un centro de referencia de alta complejidad, lo que favorece el ingreso del paciente intoxicado en diferentes estadios de su proceso fisiopatológico, incluso ya con ayudas diagnósticas realizadas previamente. El estudio es un punto de partida para investigaciones relacionadas con la pertinencia de ayudas diagnósticas en pacientes intoxicados atendidos en urgencias.

\section{DECLARACIÓN DE CONFLICTO DE INTERESES}

Los autores declaran no tener conflictos de interés.

\section{REFERENCIAS}

1. Mowry JB, Spyker DA, Brooks DE, McMillan N, Schauben JL. 2014 Annual Report of the American Association of Poison Control Centers' National Poison Data System (NPDS): 32nd Annual Report. Clin Toxicol (Phila) 2015;53:962-1147.

2. Instituto Nacional de Salud Colombia. Boletín Epidemiológico Semanal BES Semana 522018. [Internet]. [Consultado 2019 noviembre 15]. Disponible en https://www.ins.gov.co/buscadoreventos/BoletinEpidemiologico/2018\%20Bolet\%C3\%ADn\%20epidemiol\%C3\%B3gico\%20 semana\%2052.pdf

3. Instituto Nacional de Salud Colombia. Boletín Epidemiológico Semanal BES Semana 142019. [Internet] [Consultado 2019 noviembre 15]. Disponible en: https://www.ins.gov.co/buscadoreventos/BoletinEpidemiologico/2019\%20Bolet\%C3\%ADn\%20epidemiol\%C3\%B3gico\%20 semana\%2014.pdf

4. MinSalud Colombia. Intoxicaciones por sustancias químicas Colombia 2017. [Internet]. [Consultado 2019 noviembre 20]. Disponible en: https://www.ins.gov.co/buscador-eventos/ Informesdeevento/INTOXICACIONES\%202017.pdf

5. Marusich JA, Wiley JL, Lefever TW, Patel PR, Thomas BF. Finding order in chemical chaos continuing characterization of synthetic cannabinoid receptor agonists. Neuropharmacology 2018;134:73-81.

6. Murray L, Daly F, Little M, Cadogan M. Toxicology Handbook. 2. ${ }^{\text {a }}$ ed. Churchill Livingstone Australia: Elsevier Inc; 2011.

7. Albertson TE, Dawson A, De Latorre F, Hoffman RS, Hollander JE, Jaeger A, et al. TOX-ACLS: toxicologic-oriented advanced cardiac life support. Ann Emerg Med 2001;37: S78-90.

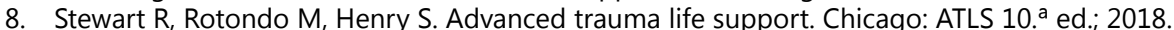

9. Brahm N, Yeager L, Fox M. False-positive urine drug screens. Ann J Health Syst Pharm 2010;67:1344-50.

10. Wallace KB, Hausner E, Herman E, Holt GD, MacGregor JT, Metz AL, et al. Serum troponins as biomarkers of drug-induced cardiac toxicity. Toxicol Pathol 2004;32(1):106-21.

11. Estrada AF, Berrouet MC, Zuluaga M, Ortiz A, Franco AK, Misas LE, et al. Epidemiología de las intoxicaciones agudas en los servicios de urgencias hospitalarias. Revista de Toxicología 2018;35:119-23.

12. Instituto Nacional de Salud Colombia. Boletín Epidemiológico Semanal BES Semana 342019. [Internet]. [Consultado 2020 agosto 12]. Disponible en: https://www.ins.gov.co/buscadoreventos/BoletinEpidemiologico/2019\%20Bolet\%C3\%ADn\%20epidemiol\%C3\%B3gico\%20 semana\%2034.pdf

13. Teran CAG, Dangond HV, Rangel AC. Análisis epidemiológico y clínico de intoxicaciones agudas atendidas en Montería, Colombia. Rev Med Risaralda 2015;21:17-21. 
14. Vallersnes $O M$, Jacobsen $D$, Ekeberg $\varnothing$, Brekke M. Patients presenting with acute poisoning to an outpatient emergency clinic: a one-year observational study in Oslo, Norway. BMC Emerg Med 2015;15:18.

15. González-Fernández D, Alonso-Fernández M. Intoxicaciones agudas en un servicio de urgencias: estudio descriptivo en el Área Sanitaria III de Asturias. Revista de Toxicología. 2009;26:122-6.

16. Zöhre E, Bozkurt S, Köse A, Demir F, Ovla D. Retrospective analysis of poisoning cases admitted to the emergency medicine. Arch Iran Med 2015;18: 117-22.

17. Goldfrank LR, Kirstein R, Goldfrank LR, Flomenbaum NE, Lewin NA, Weisman RS. Goldfrank's Toxicologic emergencies. McGraw-Hill Education; 2015.

18. Moeller KE, Kissack JC, Atayee RS, Lee KC. Clinical interpretation of urine drug tests: what clinicians need to know about urine drug screens. Mayo Clin Proc 2017:92:774-96.

19. Morris ZS, Boyle A, Beniuk K, Robinson S. Emergency department crowding: towards an agenda for evidence-based intervention. Emerg Med J 2012;29:460-6. 